\title{
A formal study of Bernstein coefficients and polynomials
}

Yves Bertot, Frédérique Guilhot and Assia Mahboubi ${ }^{\dagger}$

Received 13 December 2010

\section{Introduction}

Bernstein coefficients provide a discrete approximation of polynomials inside a bounded interval. As such they are useful tools to solve problems like locating the roots of polynomials, isolating these roots or solving systems of inequations with polynomial members. In computer aided design, they are also used intensively as they give an efficient tool to draw curves that are controlled by points that users can grab and drag, with instantaneous and intuitive feedback on the curve's shape. Bernstein coefficients are closely related to Bézier curves and they have a very simple geometrical interpretation, which we illustrate in section 4

Bernstein coefficients are defined for a given polynomial, a given degree, and a given interval. If the degree is $n$, then the coefficients form a sequence of size $n+1$. In this paper, we are interested in three important properties of these coefficients:

1 if the coefficients taken in order exhibit exactly one sign change, then the polynomial is guaranteed to have exactly one root inside the interval.

2 if all coefficients have the same sign, then the polynomial is guaranteed to have no root inside the interval.

3 there is an easy method to compute new Bernstein coefficients when splitting the interval in two.

We describe a formal proof of these three properties, concentrating on the first and third property.

The main plan of the proof of the first property is to describe a sequence of pairs $\left(I_{0}, p_{0}\right)$ to $\left(I_{3}, p_{3}\right)$, each pair containing an interval and a polynomial, such that every root of polynomial $p_{i}$ inside $I_{i}$ is in bijective correspondence with a root of polynomial $p_{i+1}$ in the interval $I_{i+1}$. If we study the roots of the polynomial $p$ on the interval $(l, r)$, then $I_{0}$ and $p_{0}$ are respectively $(l, r)$ and $p$. The last interval $I_{3}$ is $(0,+\infty)$ and the last polynomial $p_{3}$ is $c_{0}+c_{1} X+\cdots+c_{n} X^{n}$, where the coefficients $c_{i}$ have the same sign as the Bernstein coefficients. Going from $p_{i}$ to $p_{i+1}$ we apply a given transformation. The

\footnotetext{
$\dagger$ This work has been partially funded by the FORMATH project, nr. 243847, of the FET program within the 7th Framework program of the European Commission and by the Galapagos project, of the French ANR.
} 
first transformation is a change of variable such that $I_{1}$ is $(0,1)$ and $p_{1}(x)=p_{0}(r x+$ $l(1-x))$. The second transformation is such that $I_{2}$ is $(1,+\infty)$ and $p_{2}(x)=0$ exactly when $p_{1}(1 / x)=0$, as long as $x \neq 0$. The third transformation is a translation such that $I_{3}=(0,+\infty)$ and $p_{3}(x)=p_{2}(1+x)$. We will show that the condition on Bernstein coefficients simply boils down to Descartes' law of sign (Des69; BPR06) for polynomial $p_{3}$ in the case where there is exactly one sign change in this polynomial's coefficients. This path from one polynomial to another is described in section 5 .

Descartes' law of signs provides a sufficient criterion for the existence of exactly one root for a polynomial between 0 and $+\infty$. In its most general form, this law expresses a relation between the number of roots of a polynomial between 0 and $+\infty$ and the number of sign changes in the coefficients of this polynomial. The number of sign changes is larger than the number of roots and the difference between the two numbers is even. Thus, if the number of sign changes is 1 , there is exactly one root between 0 and $+\infty$.

For our development, we only prove the corollary of Descartes' law of signs for the case where there is only one sign change. This proof is done in section 3 . Expressing Descartes' law on the coefficients of polynomial $p_{3}$ yields directly a law expressed in terms of sign changes for Bernstein's coefficients of $p$ with respect to the interval $(l, r)$.

Another part of our work is to describe dichotomy. Knowing Bernstein coefficients for a polynomial and a given interval, it is easy to obtain the Bernstein coefficients for the two half intervals, using an algorithm due to de Casteljau (dC85). In the process, we increase the precision of the approximation given by the Bernstein coefficients. De Casteljau's algorithm is a simple combinatorial algorithm based on taking arithmetic means of successive coefficients. To justify this combinatorial process we show in section 4 that Bernstein coefficients actually are the coefficients of the polynomial in a different basis from the usual monomials, called the Bernstein basis.

In the following, we will assume that we are working with polynomials whose roots are all simple, called separable polynomials. Starting from an arbitrary polynomial it is easy to produce a separable polynomial with the same roots by computing the greatest common divisor of this polynomial and its derivative.

All these results deal with polynomial functions over real numbers. In order to make this formal study as generic as possible, we abstract from the choice of the subset of real numbers of interest for the user and only rely on an abstract structure of archimedean field. We however require this field to be equipped with a decidable comparison: the process we describe can in turn effectively be used in a decision procedure.

If the field we work in is only guaranteed to be ordered and archimedean, the existence of roots takes a different meaning: if a polynomial has a single simple real root in an interval, this root may not belong to the field of its coefficients. However, we can use a corresponding property which can be expressed in the field of coefficients: there exists a sub-interval inside which the absolute value of the slope is bounded below, and such that the values of the polynomial at the sub-interval bounds have opposite signs. In a similar vein, the intermediate value theorem does not hold within the language we have chosen, but a corresponding statement, expressed as a bounded-value property, does. Our proof development relies on this approach. We describe the formal aspects of this approach to describing roots in section 2 
The formal work described in this paper has been performed using the CoQ system (BC04 $\mathrm{Coq})$, with the SSREFLECT extension (GM08). We think some characteristics of the proof system played a key role in making this development possible. We describe these key aspects in section 7 . The sources of this development are available from http://hal.inria.fr/inria-00503017/

\section{Formalization viewpoints}

\subsection{A constructive and abstract approach}

Our aim is to provide a constructive and effective formalization of the theory of Bernstein polynomials, and to be as independent as possible from the implementation choices of rational numbers and real numbers. For this purpose, we eliminate real points from the formalization. We consider polynomials with coefficients in an abstract ordered and archimedean field. The axioms of this structure are the ones of a field with decidable equality, with a decidable order relation which is compatible with the field operations. The decidability of the equality and order relations are crucial for the algorithms we describe to be truely effective. Such a field necessarily contains a copy of rational numbers, which are the carrier of the computations performed in our proofs. We do not provide nor rely on a general theory of continuous functions but rather prove that such polynomials are necessarily continuous (and even uniformly continuous on bounded intervals) using $\epsilon-\delta$ statements quantified on elements of the coefficient field.

Since such an archimedean field has no reason to be algebraically closed, the roots of the polynomials are not necessarily elements of this type. We hence need to adjust the definition of root of a polynomial. In particular, we provide a weak version of the intermediate value theorem for polynomials, which results in a sufficient criterion for the existence of a single real root in an interval, expressed in the language of decidable, ordered archimedean fields. This field of coefficients cannot be directly instantiated by constructive reals since their comparison is not decidable, but this was not our purpose since our weak intermediate value theorem is only there to support the further study of Bernstein polynomials, which have rational coefficients. If coefficients are indeed instantiated by any implementation of rational numbers, for instance the one embedded in some implementation of constructive numbers, then the conclusion of the intermediate value theorem is sufficient to implement the modulus of convergence of a Cauchy sequence, which will be the constructive root of the polynomial. Of course, this work is readily usable with the "classical real numbers" of the standard CoQ library, but it also complies with the constructive libraries also implemented in the CoQ system like (CFGW04, O'C07, O'C08).

\subsection{Small scale reflection libraries}

This work is based on the SSREFLECT libraries (Mat) developed with the SSREFLECT extension (GM08) of the CoQ system. These libraries cover basic theories (sequences, natural numbers, finite types, finite sets), infrastructure for notations and theory sharing 
(containers, iterated operators, algebraic hierarchies), and elementary algebra and arithmetics. They are developed following the path leading to a complete formal proof of a historical result in finite group theory (namely the Odd Order Theorem), to demonstrate that a well understood art of formalization leads to modular, reusable formal libraries. This work addresses theories that are not on this path, it hence challenges the reusability of the distributed libraries and demonstrates that they can indeed be reused and extended.

One of the main characteristics of the methodology deployed in these libraries is small scale reflection (GM10), a proof methodology which is based on the pervasive use of computations with symbolic symbols. As a consequence, predicates are formalized as often as possible as boolean functions and a set of specialized tactics allows the user to reason on how these functions compute. For instance, it provides powerful automation for propositional reasoning: in this framework, reasoning in the intutionistically classical fragment of the CoQ logic is almost as convenient as it could be in a fully classical system. Boolean representations play a role that goes beyond automation. They also affect the manipulation of dependent types, especially dependent pairs (also called $\Sigma$-types). The SSREFLECT library on polynomials illustrate this fact as described in section 2.3 .

\subsection{Representation of polynomials}

In SSREFLECT libraries polynomials are represented by a big endian list of coefficients in the monomial basis. A polynomial function can easily be defined from such a list through the Horner evaluation scheme. A bare list of coefficients would however not provide a canonical representation since the same polynomial can be represented by an infinite number of coefficient lists, only differing by the number of tail zeroes. The actual definition is the following one:

Record polynomial $\mathrm{R}:=$

Polynomial \{polyseq :> seq $R ;$ _ : last 1 polyseq $!=0$ \}.

A polynomial is hence represented by a dependent pair consisting of a list polyseq of type (seq R) and a proof that the last element of this list is non zero (this proof is not given a name, hence the use of the character _). The type (polynomial R) is used with the notation \{poly $R$ \}. The parameter $R$ is the type of the coefficients. It should in fact be equipped with a ring structure, which provides the zero element used in the test of the proof component.

The constant zero polynomial is represented by the empty list. A constant non zero polynomial is represented by a list with a single element. The monomial $X$ is represented by the list $[0,1]$, where 1 and 0 are the one and zero constants of the underlying coefficient ring. More generally, a non zero polynomial of degree $n+1$ is represented by a list with $n+1$ elements. The :> symbol indicates that the polyseq constructor is declared as a coercion: a polynomial can at any time be seen as a list of coefficients, forgetting about the proof that it is in normal form.

In this representation, comparing two polynomials seems to amount to comparing component-wise two dependent pairs: the first components of the two pairs being the 
actual polynomials, and the second components of the pairs being the proofs that they are in normal form. The calculus of inductive constructions underlying the CoQ system is not proof irrelevant: two proofs of the same statements are not equal in general. Hence a naive design of these polynomials as dependent pairs would lead to an uncomfortable situation where two equal lists of coefficients would not necessarily give two equal polynomials because they would be associated with two different proofs of normalization. Yet if proof irrelevance does not hold in general, two proofs of the same boolean equality are nonetheless provably equal in the calculus of inductive constructions without axiom. This is known as boolean proof irrelevance.

If the type $\mathrm{R}$ of coefficients is equipped with a boolean equality test, the second component of polynomials becomes a proof that a certain boolean is equal to the boolean true, and hence a proof of a boolean equality. Comparing two polynomials now boils down to comparing only their first component, and the equality of the proof components is given by boolean proof irrelevance. This kind of proof irrelevant dependent pairs is widely used in SSREFLECT libraries, which therefore provide a generic infrastructure to manipulate comparisons of their inhabitants. Moreover, again thanks to the infrastructure of SSREFLECT libraries, polynomials canonically inherit from the boolean comparison available on coefficients, lifted to the element-wise comparison of lists.

\subsection{Criteria for the existence of a unique root}

We concentrate on a sufficient criterion for the existence of a root inside an interval. Our criterion is based on slopes.

Ensuring that the slope is positive or negative in some interval helps making sure that there are not more than one root in this interval. In our setting, where the polynomials we consider only have simple roots, we have the stronger property that the slope is separated from 0 by a given ratio. In the case of positive slopes, we write the slope requirement for a polynomial $p$ inside a given interval $I$ as follows:

Definition 2.1 (Slope requirement (positive case)). A polynomial $p$ satisfies the positive slope requirement if:

$$
\exists k,(0<k \quad \wedge \quad \forall x y,[x \in I \wedge y \in I \wedge x<y \Rightarrow k(y-x)<p(y)-p(x)])
$$

We also define the analogous requirement for negative slopes.

Depending on the kind of interval that we will consider, we will use two different sufficient conditions to express the existence of a single root in the interval.

1 If the interval is bounded, we express that the interval can be decomposed into three parts, the first part $I_{1}$ where the polynomial's value is always negative (resp. positive), the second part $I_{2}$ where the polynomial's value goes from negative to positive (resp. from positive to negative) with a requirement on the slope, and the third part $I_{3}$ where the polynomial's value is always positive (resp. negative). This is illustrated in Figure 11.

2 If the interval is unbounded, we express that the interval can be decomposed into two parts, the first part $I_{1}$ where the polynomial's value is always negative (resp. 


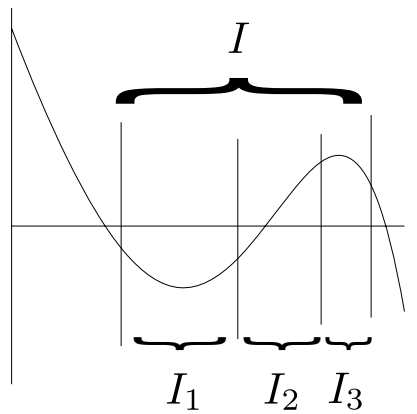

Fig. 1. A sufficient criterion for the existence of a single root in a bounded interval

positive), and the second part $I_{2}$ where there is a requirement on the slope with a positive (resp. negative) slope. This is illustrated in Figure 2

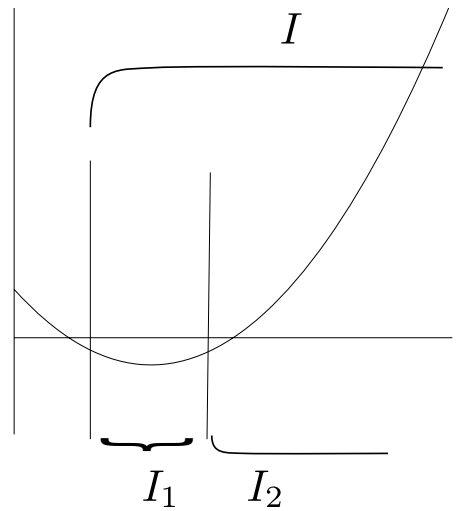

Fig. 2. A sufficient criterion for the existence of a single root in an unbounded interval

\subsection{Finding locations where a polynomial's value is arbitrarily small}

In classical mathematics dealing with real closed fields, once we know that the polynomial takes values of opposite sign at the bounds of an interval, we know that there is a root for this polynomial in this interval, thanks to the intermediate value theorem. For this work, we establish a simplified constructive, real point free, replacement of the intermediate value theorem specialized for polynomials. In our setting with a field that is only guaranteed to be ordered and archimedean, we can't be sure to produce a value on which the polynomial of interest evaluates to zero, but we are able to produce an input for which the polynomial's absolute value is arbitrarily close to zero. The statement we prove has the following form: 


\section{Theorem 2.1 (Weak intermediate value theorem for polynomials).}

$$
\begin{aligned}
& \forall p x y \varepsilon, \quad x<y \wedge 0<\varepsilon \wedge p(x)<0 \leq p(y) \\
& \quad \Rightarrow\left[\exists x^{\prime} y^{\prime},-\varepsilon \leq p\left(x^{\prime}\right)<0 \leq p\left(y^{\prime}\right) \leq \varepsilon \wedge x \leq x^{\prime}<y^{\prime} \leq y\right]
\end{aligned}
$$

Proof. We again rely on reasoning about slopes. Without loss of generality, we can assume that the two values $x$ and $y$ are positive. Assuming that the polynomial $p$ has the shape $a+X \times p_{1}$, we construct another polynomial $p_{2}$ whose coefficients are the absolute values of the coefficients of $p_{1}$. This polynomial $p_{2}$ is increasing so its maximum value in $[x, y]$ is reached in $y$. We prove that the slope of the polynomial between any two points inside $[x, y]$ is smaller than $k=p_{2}(y)$. Thus, we establish that the slope of any polynomial is bounded in absolute value on any bounded interval. In particular, for any $z, t \in[x, y]$, we have

$$
|p(z)-p(t)| \leq|k \times(z-t)| .
$$

For a given $\varepsilon$, because we work in an archimedean field, we can choose an $n$ such that $\frac{k(y-x)}{n}<\varepsilon$ We then consider the $n+1$ values $a_{i}=x+\frac{i \times(y-x)}{n}$ for $i=0 \ldots n$ and we solve a discrete problem over the values $a_{i}$. We simply need to find the largcmpest prefix $a_{0}, \ldots, a_{j-1}$ such that all values $p\left(a_{k}\right)$ in this prefix are negative. We can set $x^{\prime}=a_{j-1}$, because the next value $p\left(a_{j}\right)$ is necessarily non-negative and $p\left(a_{j}\right)-p\left(x^{\prime}\right)<\varepsilon$, thus $-\varepsilon<p\left(x^{\prime}\right)<0$. In a similar way, we can set $y^{\prime}=a_{j}$ because $0 \leq p\left(y^{\prime}\right)<\varepsilon$.

Our algorithm is illustrated in Figure 3 . The point selected by our algorithm is $a_{8}$, even though there are more roots in the vicinity of $a_{1}$ and $a_{2}$ but neither $a_{1}$ nor $a_{2}$ is a point where the polynomial takes a positive value.

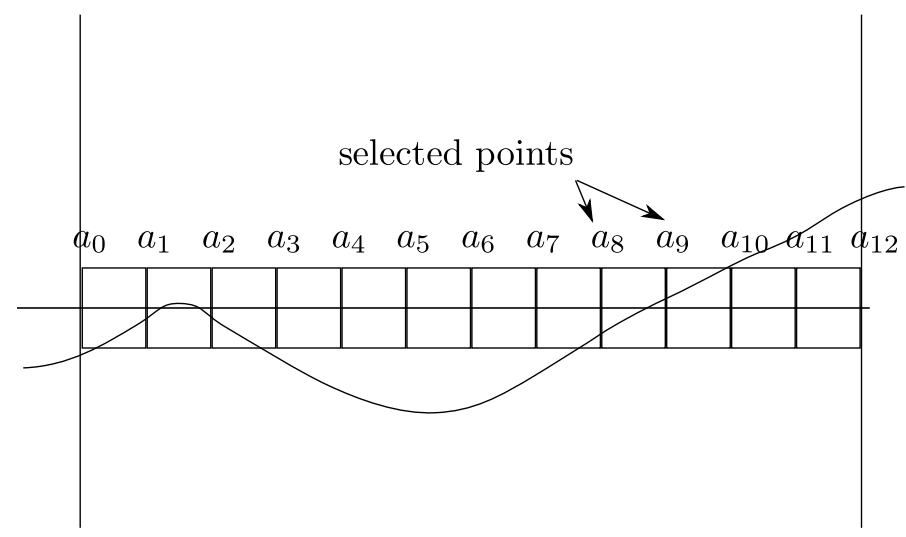

Fig. 3. Bounding a polynomial's value

This theorem provides a similar result to theorem 6.1.4 from (TvD88), but the proof in that book relies on a more complete description of abstract topology than we performed of our development, where opens and their properties with respect to continuous functions are not mentioned. The next theorem 6.1.5 from (TvD88) makes it possible to construct a Cauchy sequence to a root of the continuous function being considered. A variant of our theorem would also make this possible, but this was not needed for our purposes. 


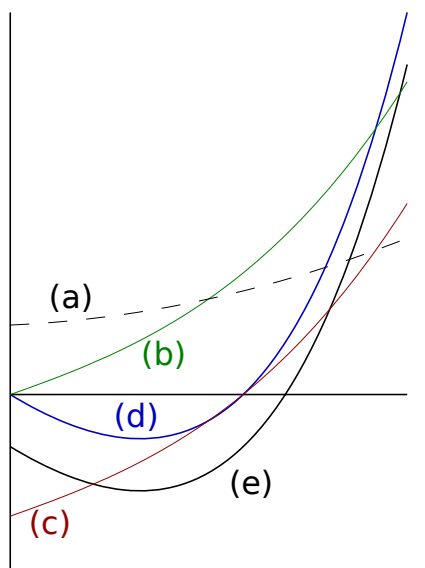

(a) non-negative coefficients and a non-zero constant coefficient,

(b) non-negative coefficients and a zero constant coefficient,

(c) a negative constant coefficient and all others non-negative,

(d) one sign change and a zero constant coefficient,

(e) one sign change and a negative constant coefficient

Fig. 4. Classes of polynomials with or without sign change

\section{A simple form of Descartes' law of signs}

One of the main results studied in this paper is that having only one sign change in the sequence of Bernstein coefficients for the square-free polynomial $p$ and the interval $(l, r)$ ensures that there is only one root of $p$ inside $(l, r)$. The proof of this result relies on a similar property for the standard coefficients of another polynomial $q$ : if there is only one sign change in the coefficients of $q$ then $q$ has only one root inside the interval $(0,+\infty)$. In this section, we discuss how this property is proved formally.

\subsection{A Geometrical explanation of the proof}

Let's first describe a simple graphical argument based on curves for polynomial functions between 0 and $+\infty$, as shown in Figure 4 . To describe our proof, we rely on an inductive view of polynomials where new polynomials are built from existing ones by multiplying them by the polynomial $X$ and adding a constant; this operation is known as "Horner's scheme". Polynomials with one sign change and a positive leading coefficient are obtained by starting with a positive constant, applying Horner's scheme a certain number of times with non-negative constants, then applying it with a negative constant, and then applying it again a certain number of times with non-positive constants.

Polynomials with only non-negative coefficients have curves which look like the curves (44a) or (4-b) depending on whether the first coefficient is 0 , adding a positive coefficient to a polynomial of the form (44a) or (4 b) yields a polynomial of the form (4 a), multiplying a polynomial of the form (4 a) or (4 b) by the polynomial $X$ yields a new polynomial of the form (4-b). Thus, Horner's scheme with non-negative constants keeps polynomials 
in the (a-b) form. Then, when applying Horner's scheme with a negative coefficient (thus introducing a sign change), the multiplication by $X$ first builds a polynomial of the (4-b) form, and adding a negative constant, one obtains a curve whose shape is given by (4 c). From then on, multiplying a polynomial of the form (4 $\mathrm{c}$ ), (4 $\mathrm{f}$ ), or (4 e) by $X$ yields a polynomial of the $(4 \mathrm{~d})$ form; adding a negative constant to a polynomial of the form (4t d) or (4 e) yields a polynomial of the (4 -e) form. Polynomials of the form (4 $\mathrm{d}$ ) or (4 e) share the following characteristic: there exists a positive value $x$, such that the polynomial has a negative value between 0 and $x$, and the slope of the curve is strictly positive above $x$. Because of the slope condition, we can also find a point where the polynomial is positive.

Let us now give a more precise proof, outlining the concepts that are used in the formal proof.

\subsection{Lemmas for polynomials with non-negative coefficients}

Polynomials are simply encoded by their lists of coefficients, evaluating a polynomial at a given point is done recursively following Horner's scheme, and recognizing polynomials with only non-negative coefficients is also done using a simple recursive function, written in the following form:

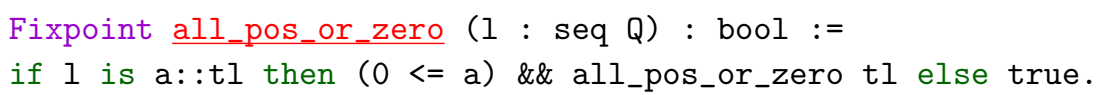

The type $\mathbf{Q}$ refers to the ordered field parameterizing the development, $(0<=a)$ is a boolean value, and the type constructor seq is a type for lists of elements in a type equipped with a boolean equality test.

We should notice that polynomials satisfying the boolean predicate all_pos_or_zero may contain no positive coefficients: for this reason, we cannot guarantee that they are increasing or strictly positive anywhere between 0 and $+\infty$.

We prove easily by induction on lists that if they contain only non-negative coefficients, then the corresponding polynomial always has a non negative value for inputs in $(0,+\infty)$ and from then, we also prove by induction that any polynomial with only non-negative coefficients is constant or increasing.

We then prove that for every polynomial $p$ with non-negative coefficients, the product $x \times p(x)$ can be made arbitrarily close to 0 while $x$ stays in $(0,+\infty)$.

\subsection{Two lemmas on slopes}

A first lemma on slopes concerns the existence of points where a polynomial $p$ takes a value above an arbitrary bound $a$. If the slope is bounded below by a positive ratio $k$, this is guaranteed as it suffices to take an input that is large enough. As the proof is constructive, we need to be more precise: assuming the slope is larger than $k$ for any $y$ larger than $x_{1}$, it suffices to take any input larger than $x_{1}+\frac{a-p\left(x_{1}\right)}{k}$. This result is remembered in our development under the name above_slope.

A second lemma on slopes concerns the slope of a product of the form $x \times p(x)$. 
This lemma reproduces the known formulas for the derivative of products of derivable functions, but is expressed solely in terms of lower bounds of slopes: If a function $f$ has a slope larger than or equal to a non-negative ratio $k_{f}$ when $x$ is larger than a certain bound $a$, then the slope of the product $x \times f(x)$ is larger than $a k_{f}+f(x)$.

This statement requires $f$ to have a positive slope, but it leaves open whether $f(x)$ is positive or not. In particular, the values $a$ and $k_{r}$ can be fixed for a large interval: we intend $a$ to be the lower bound of interval $I_{2}$ as used in the criterion for existence of a unique root in an unbounded interval (see Figure 2).

\subsection{Polynomials with exactly one sign change}

We can now address the case of polynomials with exactly one sign change. We want to show that these polynomials have exactly one root. We exhibit the two intervals described in the criterion for unbounded intervals (see Figure 2 the positive value $x_{1}$ and the positive ratio $k$ such that the polynomial is negative in the interval $\left(0, x_{1}\right)$ and the slope between any two values above $x_{1}$ is larger than $k$.

To detect polynomials with exactly one sign change, we use two recursive functions. The first one, which we call alternate_1, recognizes polynomials with at least one positive coefficient, preceded by any number of non-positive coefficients (possibly 0), and followed by only non-negative coefficients, as checked by all_pos_or_zero. This function is defined as follows:

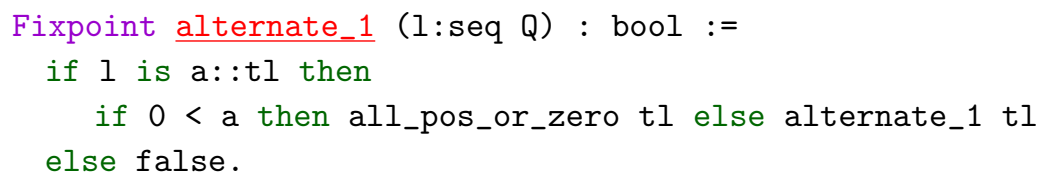

The second function, which we call alternate, checks for the presence of at least one negative coefficient and then calls alternate_1.

As we have two recursive functions, alternate_1 and alternate, we actually need to perform two proofs by induction. Each proof by induction shows that some invariant is satisfied.

The invariant for alternate_1 must be satisfied by a polynomial $p$ that may or may not contain a negative coefficient, so that this invariant cannot guarantee the existence of places where the polynomial takes a negative value. Instead, this invariant guarantees for any positive $\varepsilon$ the existence of a positive $\mathrm{x}$ and a $k$ such that:

1 for any $y$ between 0 and $x, p(y) \leq p(x)$,

2 the slope between two points larger than $x$ is guaranteed to be larger than $k$,

3 the number $x \times p(x)$ is between 0 and $\varepsilon$.

The invariant for alternate is exactly the criterion we use to describe the existence of exactly one root in an unbounded interval as in section 2.4. This proof by induction is done by induction on the list. The empty list does not satisfy the predicate alternate so that this case is easily taken care of. The other case is when the polynomial is described by a list of the form $a:: l$, so that $l$ represents another polynomial $p_{l}$ and $p(x)=a+x \times p_{l}(x)$. Here another case distinction must be studied, depending on whether $a$ is zero or negative. 
If $a$ is negative, we cannot use an induction hypothesis, because in this case $l$ is only guaranteed to satisfy the predicate alternate_1. On the other hand, the invariant for alternate_1 guarantees the existence of an $x$ such that $x \times p_{l}(x)$ is positive and smaller than $-a$, this $x$ is the right witness and the slope is $p_{l}(x)$. Since $p_{l}(y) \leq p_{l}(x)$ when $y<x$ it is easy to prove that $a+y \times p_{l}(y)=p(y)$ is negative when $0<y \leq x$. To reason on the slope, we use our lemma about the slope of $x \times p_{l}(x)$, using 0 as a lower bound for the slope of $p_{l}$ (we only know that it is increasing).

If a is zero, we have by induction hypothesis that there exist $x$ and $k$ such that $p_{l}$ is negative on the left of $x$ and has a slope larger than $k$ on the right of $x$. However, this does not guarantee that $x$ is the right witness for $p$ because the slope of $x \times p_{l}(x)$ is only larger than $p_{l}(x)+x \times k$, and $p_{l}(x)$ is negative. The solution is to note that $p_{l}$ necessary takes a positive value in some point $v_{1}$ on the right of $x$ and to use our constructive intermediate value theorem from section 2.5 to build a new value $x_{1}$ such that $-\frac{k v_{1}}{2} \leq p_{l}\left(x_{1}\right) \leq 0$ and $x_{1}<v_{1}$. Now $p_{l}$ is still guaranteed to be negative between $x$ and $x_{1}$, because of the slope condition, and the slope on the right of $x_{1}$ is guaranteed to be larger than $\frac{k v_{1}}{2}$, which is positive.

\section{Bernstein polynomials, Bernstein coefficients}

Bézier curves (Béz86) are parametric curves that are widely used to construct smooth plane curves whose shapes are governed by a finite finite number of control points. A Bézier curve controlled by $n+1$ points is a polynomial expression of degree $n$ in its parameter $t$. For instance, given two points $P_{0}$ and $P_{1}$, the corresponding Bézier curve is the segment $B_{\left(P_{0}, P 1\right)}(t)=t P_{0}+(1-t) P_{1}$. For three control points $P_{0}, P_{1}, P_{2}$, the Bézier curve is $B_{\left(P_{0}, P_{1}, P_{2}\right)}(t)=(1-t)^{2} P_{0}+2(1-t) t P_{1}+t^{2} P_{2}$. We already see in this case that a Bézier curve does not meet all its control points. In fact, it is only guaranteed to pass through the first and the last control point. In the case of the quadratic Bézier curve, the middle control point $P_{1}$ is the intersection of the tangents to the curve at $P_{0}$ and $P_{2}$. The general formula giving the Bézier curve with $n+1$ control points is:

$$
B_{\left(P_{0}, \ldots, P_{n}\right)}(t)=\sum_{k=0}^{n}\left(\begin{array}{l}
n \\
k
\end{array}\right)(1-t)^{n-k} t^{k} P_{i}
$$

which satisfies the recursive relation:

$$
B_{\left(P_{0}, \ldots, P_{n}\right)}(t)=(1-t) B_{\left(P_{0}, \ldots, P_{n-1}\right)}(t)+t B_{\left(P_{1}, \ldots, P_{n}\right)}(t)
$$

Bézier curves are named after the engineer Paul Bézier who was working in the design division of a car manufacturing company. These curves have very interesting properties for interpolation but also for computer graphics: a Bézier curve is contained in the convex hull of its control points and uniform transformations on the control points like translation or rotation have the same effect on the curve. Points control the shape of the curve since the $k$-th derivative of the curve at its extreme points is governed by the $k+1$ nearest control points.

Computer graphics algorithms usually use piecewise polynomial paths (called splines) 
of low degree. Bézier curves are often used to build these splines, resulting in the socalled Bézier splines. Most modern vector graphics standards, like for instance SVG, feature support for Bézier splines. TrueType fonts use quadratic Bézier splines, whereas PostScript or MetaFont (Knu86) use cubic Bézier splines.

Bernstein polynomials are defined as the weight assigned to each control point: the $k$-th Bernstein polynomial $P_{b}(n, k)$ is defined by:

$$
B_{\left(P_{0}, \ldots, P_{n}\right)}(t)=\sum_{k=0}^{n} P_{b}(n, k)(t) P_{k}
$$

hence:

$$
P_{b}(n, k)(t)=\left(\begin{array}{l}
n \\
k
\end{array}\right)(1-t)^{n-k} t^{k}
$$

For arbitrary numbers $l$ and $r$, we can also consider the following polynomials, called the Bernstein polynomials for degree $n$ and the interval $(l, r)$ for $0 \leq k \leq n$

$$
P_{b}(n, l, r, k)=\left(\begin{array}{l}
n \\
k
\end{array}\right) \frac{(x-l)^{k}(r-x)^{n-k}}{(r-l)^{n}}
$$

These polynomials also constitute a basis of the vector space of polynomials of degree $n$, and we will usually call it the Bernstein basis leaving the degree and the values $l$ and $r$ unspecified. Every polynomial $p$ of degree $n$ hence has a sequence of coefficients $\left(b_{i}\right)_{0 \leq i \leq n}$, such that $p(x)=\sum_{i=0}^{n} b_{i} P_{b}(n, l, r, i)(x)$. The coefficients $b_{i}$ are the Bernstein coefficients of $p$.

When $l<r$, the Bernstein polynomials are positive on $(l, r)$ and each polynomial of index $k$ reaches its maximum at the point $d_{k}=l+\frac{(r-l) k}{n}$, so that each coefficient $b_{k}$ somehow has a dominant influence on the value of the polynomial around $d_{k}$. Moreover, the coefficients $\left(\begin{array}{l}n \\ k\end{array}\right)$ included in the definition of $P_{b}(n, l, r, k)$ are chosen in such a way that the $k$-th coefficient $b_{k}$ of $p$ would tend to have a value close to the one of $p$ in $d_{k}$. For instance, if $p_{1}$ is the constant polynomial with value 1 , then all its Bernstein coefficients are equal to 1 ; similarly, if $p_{2}$ is the identity polynomial, and $n$ is larger than 0 , then the Bernstein coefficients for $p_{2}$ are $l+\frac{(r-l) k}{n}$, as can be verified using the following computation:

$$
\begin{aligned}
\sum_{i=0}^{n} & \left(l+\frac{(r-l) i}{n}\right)\left(\begin{array}{c}
n \\
i
\end{array}\right) \frac{(x-l)^{i}(r-x)^{n-i}}{(r-l)^{n}} \\
& =\sum_{i=0}^{n} l\left(\begin{array}{c}
n \\
i
\end{array}\right) \frac{(x-l)^{i}(r-x)^{n-i}}{(r-l)^{n}}+\sum_{i=0}^{n} \frac{i}{n}\left(\begin{array}{c}
n \\
i
\end{array}\right) \frac{(x-l)^{i}(r-x)^{n-i}}{(r-l)^{n-1}} \\
& =l \frac{((x-l)+(r-x))^{n}}{(r-l)^{n}}+\sum_{i=1}^{n}\left(\begin{array}{c}
n-1 \\
i-1
\end{array}\right) \frac{(x-l)^{i}(r-x)^{n-i}}{(r-l)^{n-1}} \\
& =l+(x-l) \sum_{i=0}^{n-1}\left(\begin{array}{c}
n-1 \\
i
\end{array}\right) \frac{(x-l)^{i}(r-x)^{(n-1)-i}}{(r-l)^{n-1}} \\
& =l+(x-l) \frac{((x-l)+(r-x))^{n-1}}{(r-l)^{n-1}}=x
\end{aligned}
$$




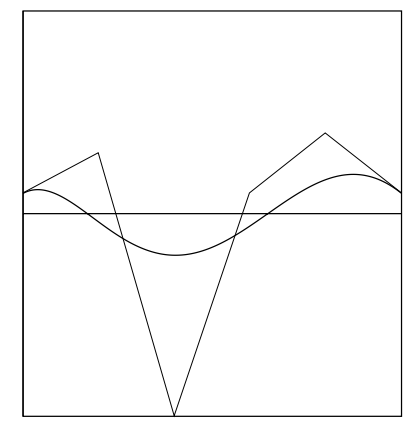

(a) $1,3,-10,1,4,1$

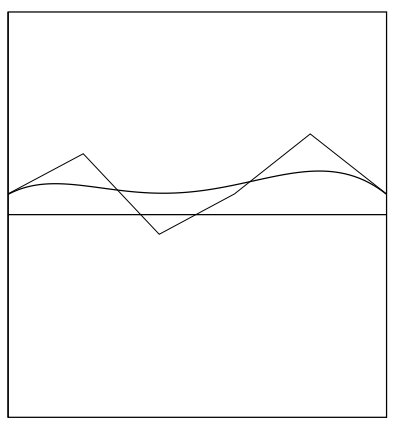

(b) $1,3,-1,1,4,1$

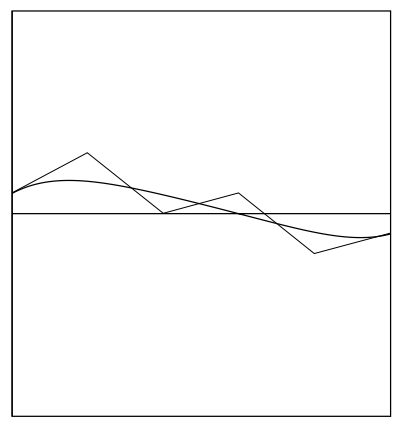

(c) $3,0,1,10,-2,-1$

Fig. 5. Bernstein Control points and corresponding polynomial curves

At the first equality sign, we distribute inside the first sum; in the second term, we simplify the denominator with the numerator $(r-l)$. At the second equality sign, we recognize that the first term contains a binomial formula corresponding to $((x-l)+(r-x))^{n}$; in the second term, we recognize that the first element of the sum can be removed because it is 0 , also we recognize that $\frac{i}{n}\left(\begin{array}{c}n \\ i\end{array}\right)$ is $\left(\begin{array}{c}n-1 \\ i-1\end{array}\right)$ when $i \neq 0$. At the third equality sign, we use the equality $(x-l)+(r-x)=r-l$ for the first term and we factor out $(x-l)$ from the remaining indexed sum and re-index that sum. We then recognize another binomial formula and can conclude.

The Bernstein coefficients are related to a broken line (made of contiguous straightline segments) which gives a rough approximation of the polynomial's function graph. More precisely, given the bounds $(l, r)$ of the interval, and the Bernstein coefficients $\left(b_{0}, \ldots, b_{n}\right)$ of polynomial $p$, the $n+1$ points with coordinates $\left(l+i \frac{r-l}{n}, b_{i}\right)$ are the control points for the Bézier curve that coincides with the polynomial's graph. This is illustrated in Figure 5

In Figure (5-a) the illustration shows that a peak in the disposition of the control points corresponds to an inflexion in the polynomial's curve (the Bernstein coefficients are 1, 3, $-10,1,4,1$ and -10 corresponds to a downward peak). In this case, the peak provokes two sign changes, which are reproduced in the shape of the curve and correspond to the existence of two roots inside the interval. In Figure (5-b), the coefficients still exhibit a downward peak with a negative coefficient, but the polynomial's curve stays away from the $\mathrm{x}$-axis and the two sign changes in the Bernstein coefficients do not correspond to any real root for the polynomial (this is a false alert). In Figure (5.c), there is one sign change, so that the first and last coefficients have opposite signs. In fact, the first and the last Bernstein coefficients are equal to the values of the polynomial at the bounds of the interval, so this imposes the existence of at least one root. But because there is exactly one sign change in the coefficients, we can be sure that any other bend in the curve stays away from the $\mathrm{x}$-axis. 


\section{From Bernstein to Descartes}

In this section, we clarify the polynomial transformations that link the problem of finding the roots of a polynomial inside an arbitrary bounded interval $(l, r)$ successively with the problem of finding the roots of another polynomial inside the interval $(0,1)$ and with the problem of finding the roots of yet another polynomial inside the interval $(0,+\infty)$. These transformations make it possible to compute another collection of coefficients, which happen to be very simply related to Bernstein coefficients.

\subsection{A criterion for the interval $(1,+\infty)$}

Descartes' weak law of signs gives us a sufficient condition to determine when the unbounded interval $(0,+\infty)$ contains exactly one root for a polynomial. Through a change of variable, we obtain a similar criterion for the interval $(1,+\infty)$.

In the following, we will call $\theta_{v}$ the transformation that maps any polynomial $p$ to the polynomial $y \mapsto p(y+v)$. If $p=\sum_{i=0}^{n} a_{i} x^{i}$, we have the following formula:

$$
p(y+v)=\sum_{i=0}^{n} a_{i}(y+v)^{i}=\sum_{k=0}^{n}\left(\sum_{i=k}^{n} a_{i}\left(\begin{array}{l}
i \\
k
\end{array}\right) v^{i-k}\right) y^{k}
$$

The polynomial $p$ has exactly one root in the interval $(v,+\infty)$ if and only if the polynomial $\theta_{v}(p)$ has exactly one root in the interval $(0,+\infty)$. We proved this lemma.

Thus, if we apply Descartes's law of signs on the coefficients $\sum_{i=k}^{n} a_{i}\left(\begin{array}{l}i \\ k\end{array}\right)$ we can obtain a sufficient criterion for the existence of exactly one root of the polynomial $p=\sum_{i=0}^{n} a_{i} x^{i}$ in the interval $(1,+\infty)$.

\subsection{A criterion for the interval $(0,1)$}

Descartes' law of signs works for unbounded intervals. In this section, we see how to cover also bounded intervals. The trick here relies on reversing the polynomial's list of coefficients. Obviously, the number of sign changes in a list of coefficients is the same as the number of sign changes in the reversed list.

However, the roots of a polynomial on the interval $(1,+\infty)$ are in one-to-one correspondence with the roots of the reversed polynomial in $(0,1)$. This is due to the following equation:

$$
\sum_{i=0}^{n} a_{i} x^{i}=x^{n} \times \sum_{i=0}^{n} a_{i} x^{i-n}
$$

We can now perform another change of variable, here $y=1 / x$ and a change of index $j=n-i$ in the sum.

$$
\sum_{i=0}^{n} a_{i} x^{i}=\left(\frac{1}{y}\right)^{n} \sum_{j=0}^{n} a_{n-j} y^{j}
$$

The polynomial $\sum_{j=0}^{n} a_{n-j} y^{j}$ is exactly the reversed polynomial in $y$, and the expression $\left(\frac{1}{y}\right)^{n}$ never becomes 0 for $y \in(0,1)$. Thus, $x$ is a root of the polynomial between 1 and $+\infty$ if and only if $y=x^{-1}$ is a root of the reversed polynomial between 0 and 1 . 


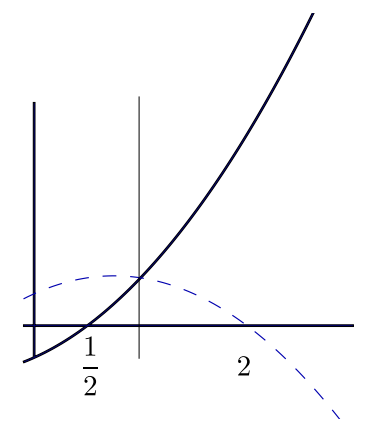

Fig. 6. Curves of $x^{2}+\frac{3}{2} x-1$ (solid line) and its reverse $-x^{2}+\frac{3}{2} x+1$ (dashed)

Let us note $\rho$ the function that computes the reverse of a polynomial. Here we need to be precise: the coefficients of a polynomial of degree $n$ actually are the coefficients of a vector in an $n+1$ dimensional space, whose basis is made of the monomials $X^{i}$ where $i \in\{0, \ldots, n\}$. Seen as an operation on this vector space, $\rho$ is an involutive automorphism. But polynomials of degree less than $n$ are also elements of this vector space and the reverse operation must be understood as reversing the list of coefficients of length $n+1$ obtained by padding the polynomials description with enough 0 coefficients.

To illustrate the correspondence between a polynomial and its reverse, we can consider the polynomial $p(x)=x^{2}+\frac{3}{2} x-1$, the reversed polynomial is $q(x)=-x^{2}+\frac{3}{2} x+1$ and after the variable change we obtain the polynomial $-x^{2}-2 x+1$ which exhibits only one sign change. This predicts that the polynomial has exactly one root between 0 and 1 , and indeed the two roots of the initial polynomial are -2 and $1 / 2$. This is illustrated in Figure 6 where the curve with a solid line is the curve for the polynomial $p$, while the curve with a dashed line is the curve for the polynomial $q$, which has a single root between 1 and $+\infty$.

As a conclusion, we can establish a correspondence between unique roots in $(1,+\infty)$ of a polynomial $p$ and unique roots in $(0,1)$ of the polynomial $\rho(p)$. When working in a field that is only guaranteed to be ordered and archimedean, this correspondence works by linking the criterion for unbounded intervals with the criterion for bounded intervals. The proof of this correspondence involves the computation of the slope for $x^{n} p(1 / x)$ from the slope of $p$, which makes it trickier than other proofs of this section. This is again a place where our constructive intermediate value theorem plays a role.

\subsection{Handling arbitrary bounded intervals}

The next step is to relate the roots of any polynomial inside an arbitrary interval $(l, r)$ with the roots of another polynomial inside the interval $(0,1)$. This is done with another change of variable, this time $x=(r-l) y+l$. In other words, the polynomial function which maps any $x$ to $p(x)$ has a root between $l$ and $r$ if and only if the polynomial function which maps any $y$ to $p((r-l) y+l)$ has a root between 0 and 1 .

Here again, we can define a generic transformation on polynomials, named $\chi_{k}$ that corresponds to expanding with a given ratio $k$. For an arbitrary polynomial $p=\sum_{i=0}^{n} a_{i} X^{i}$, 


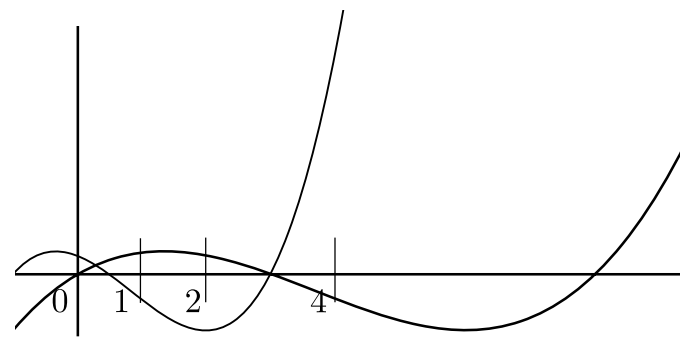

Fig. 7. Curves of a polynomial inside $(2,4)$ and the corresponding transformed polynomial inside $(0,1)$

the polynomial $\chi_{k}(p)$ is defined as follows:

$$
\chi_{k}(p)=\sum_{i=0}^{n} a_{i}\left(k X^{i}\right)=\sum_{i=0}^{n} a_{i} k^{i} X^{i}
$$

Thus, the change of variable to study the roots of polynomial $p$ is actually represented by $\chi_{r-l} \circ \theta_{l}$.

The geometric effect of the polynomial transformation is illustrated in Figure 7 , where the shape of the curve for the polynomial $\frac{x^{3}}{8}-\frac{x^{2}}{8}+3 x$ inside the interval $(2,4)$ is reproduced by the shape of the curve for the polynomial $x^{3}-\frac{5}{2} x^{2}-2 x+\frac{3}{2}$ inside the interval $(0,1)$.

\subsection{Recapitulating operations}

In our formal development, we defined the three operations for translating $(\theta)$, expanding $(\chi)$, and reversing the list of coefficients $(\rho)$. We can then compute a sequence of coefficients by applying the transformation

$$
\tau=\theta_{1} \circ \rho \circ \chi_{r-l} \circ \theta_{l} .
$$

When the coefficients we obtain have exactly one sign change, we know that the polynomial has exactly one root inside the interval $(l, r)$.

By construction, each of the operation $\theta, \rho, \chi$ actually is a linear application of the vector space of polynomials of degree less than $n$ into itself. The inverse of $\theta_{a}$ is $\theta_{-a}$, and this is easily proved, so that $\theta_{a}$ is always bijective. When $k$ is nonzero, the inverse of $\chi_{k}$ is $\chi_{\frac{1}{k}}$, so this linear application is also bijective. The inverse of $\rho$ is itself. As a result, the whole transformation is also inversible and its inverse is

$$
\tau^{-1}=\theta_{-l} \circ \chi_{\frac{1}{r-l}} \circ \rho \circ \theta_{-1} .
$$

We proved that the images of the monomials $X^{i}$ by this inverse transformation are multiples of the Bernstein polynomials, in reverse order. Let us first observe the effect of 
$\rho \circ \theta_{-1}$ :

$$
\begin{aligned}
\tau^{-1}\left(x^{i}\right)=\theta_{-l} \circ \chi_{\frac{1}{r-l}} \circ \rho\left(\sum_{j=0}^{i}\left(\begin{array}{l}
i \\
j
\end{array}\right)(-1)^{j} x^{i-j}\right) & =\theta_{-l} \circ \chi_{\frac{1}{r-l}}\left(\sum_{j=0}^{i}\left(\begin{array}{l}
i \\
j
\end{array}\right)(-1)^{j} x^{n-i+j}\right) \\
& =\theta_{-l} \circ \chi_{\frac{1}{r-l}}\left(x^{n-i} \sum_{j=0}^{i}\left(\begin{array}{l}
i \\
j
\end{array}\right)(-x)^{j}\right) \\
& =\theta_{-l} \circ \chi_{\frac{1}{r-l}}\left(x^{n-i}(1-x)^{i}\right)
\end{aligned}
$$

Then let's observe the effect of $\theta_{-l} \circ \chi_{\frac{1}{r-l}}$.

$$
\begin{aligned}
\tau^{-1}\left(X^{i}\right) & =\theta_{-l}\left(\frac{x^{n-i}}{(r-l)^{n-i}}\left(1-\frac{x}{r-l}\right)^{i}\right) \\
& =\theta_{-l}\left(\frac{x^{n-i}}{(r-l)^{n-i}} \frac{(r-l-x)^{i}}{(r-l)^{i}}\right) \\
& =\theta_{-l}\left(\frac{x^{n-i}(r-l-x)^{i}}{(r-l)^{n}}\right) \\
& =\frac{(x-l)^{n-i}(r-l-(x-l))^{i}}{(r-l)^{n}} \\
& =\frac{1}{\left(\begin{array}{c}
n \\
i
\end{array}\right)}\left(P_{b}(n, l, r, n-i)\right)
\end{aligned}
$$

If the transformation $\tau(p)$ leads to a sequence of coefficients $c_{i}$, this means $\tau(p)=$ $\sum_{i=0}^{n} c_{i} x^{i}$. Now, using the fact that both $\tau$ and $\tau^{-1}$ are linear, we can see that the polynomial $p$ is

$$
\begin{aligned}
p & =\tau^{-1}\left(\sum_{i=0}^{n} c_{i} X^{i}\right) \\
& =\sum_{i=0}^{n} c_{i} \tau^{-1}\left(x^{i}\right) \\
& =\sum_{i=0}^{n} c_{i} \frac{1}{\left(\begin{array}{c}
n \\
i
\end{array}\right)} P_{b}(n, l, r, n-i)
\end{aligned}
$$

Thus, the Bernstein coefficients are obtained in the following manner:

$$
b_{i}=\left(\begin{array}{c}
n \\
n-i
\end{array}\right)^{-1} c_{n-i}=\left(\begin{array}{c}
n \\
i
\end{array}\right)^{-1} c_{n-i}
$$

Since the number of sign changes does not depend on the order in which the list is observed, we obtain the proof that one sign change in the sequence of Bernstein coefficients implies the existence of a root in the interval $(l, r)$. 


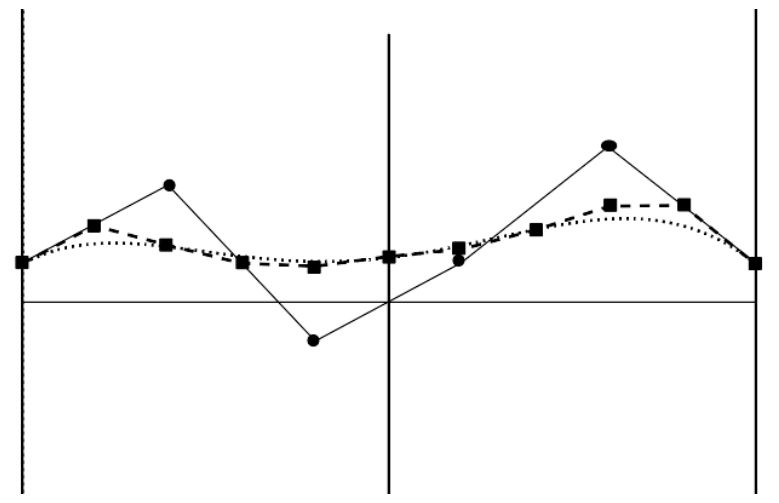

Fig. 8. Bernstein control points for halved intervals

\section{Dichotomy}

Bernstein coefficients give precise information when they exhibit either zero or one sign change. In the first case, we have the guarantee that there are no roots of the considered polynomial in the considered interval. In the second case, we have the guarantee that there is exactly one root.

When Bernstein coefficients exhibit more than one sign change, no conclusion can be drawn about the existence and unicity of roots in the interval. For instance, in Figure $(5 \mathrm{~b})$, the Bernstein coefficients exhibit two sign changes, but there is no root inside the interval. When facing this kind of inconclusive information, the solution is to refine the approximation given by the control line.

\subsection{Geometric intuition for dichotomy}

When cutting an interval in two halves, the number of control points is approximately doubled, because each of the new half-intervals receives a new sequence of $n$ Bernstein coefficients. As a result, the control points are closer to each other and to the polynomial's curve and they give a more accurate account of the curve's position with respect to the real x-axis. This is illustrated in Figure 8, where the initial Bernstein coefficients exhibit two sign changes, which are needed to account for the bend in the first half of the interval (a positive local minimal, but expressed by a negative Bernstein coefficient). In the halved interval two more points are added in the vicinity of the bend, and none of the control points have to be negative anymore.

In Figure 8, the dotted line represents the polynomial's curve, the solid line links the control points for the largest interval, marked by round bullets (the Bernstein coefficients are 1, 3, -1, 1, 4, 1 for this interval). The dashed line links the control points for the two half intervals, marked by square boxes (the Bernstein coefficients are 1, 2, 1.5, 1, 0.9375, 1.15625 for the first interval, and 1.15625, 1.375, 1.875, 2.5, 2.5, 1 for the second interval). This figure illustrates that the control line really gets closer to the polynomial's curve, and provides a much better approximation of the polynomial.

The formula given in section 4 is useful to compute an initial series of Bernstein 
coefficients, and the correctness of the conditions for existence of roots based on these coefficients can be justified using the transformation described in section 5.4

It may seem that computing Bernstein coefficients is a costly process. Around 1950, while studying Bézier curves, De Casteljau noticed that the coefficients for the sub intervals were easy to compute from the coefficients for the big interval through a simple recursive process, exploiting the recurrence relation already given in section 4 . This suggests another recursive algorithm, starting from a large interval that is guaranteed to contain all the roots of a polynomial (see section 6.2 and splitting this interval into smaller pieces until all roots have been isolated (see section 6.3).

\subsection{Initialization}

Given an arbitrary non constant polynomial $p$ of degree $n$, defined by $p=\sum_{i=0}^{n} a_{i} X^{i}$ it is actually possible to bound the absolute values of its roots by a simple constant defined from the coefficients $a_{i}(i=0 \ldots n)$, called the Cauchy bound (BPR06):

\section{Theorem 6.1 (Cauchy bounds).}

$$
\forall x \in \mathbb{R}, p(x)=0 \Rightarrow|x| \leq C(p) \quad \text { with } \quad C(p)=\sum_{i=0}^{n} \frac{\left|a_{i}\right|}{\left|a_{n}\right|}
$$

Proof. Let $x$ be a root of $p$. If $|x| \leq 1$, since $1 \leq C(p)$, the inequality trivially holds. Then if $|x|>1$, since $x$ is a root, and $a_{n} \neq 0$

$$
x^{n}=-\frac{1}{a_{n}} \sum_{i=0}^{n-1} a_{i} x^{i}
$$

Hence:

$$
|x|^{n} \leq \frac{1}{\left|a_{n}\right|} \sum_{i=0}^{n-1}\left|a_{i}\right||x|^{i} \leq \frac{1}{\left|a_{n}\right|} \sum_{i=0}^{n}\left|a_{i}\right||x|^{i}
$$

by triangular inequality. Then:

$$
|x| \leq \frac{1}{\left|a_{n}\right|} \sum_{i=0}^{n-1}\left|a_{i}\right||x|^{i-(n-1)} \leq \frac{1}{\left|a_{n}\right|} \sum_{i=0}^{n-1}\left|a_{i}\right|
$$

since $|x|>1$ implies that forall $i=0 \ldots n-1,|x|^{i-(n-1)} \leq 1$. Finally since:

$$
\frac{1}{\left|a_{n}\right|} \sum_{i=0}^{n-1}\left|a_{i}\right| \leq \frac{1}{\left|a_{n}\right|} \sum_{i=0}^{n}\left|a_{i}\right|
$$

we have $|x| \leq C(p)$.

This means that to start studying the roots of a polynomial $p$ we can restrict the infinite real line to a bounded interval $(-C(p), C(p))$. This justifies we can start a real root isolation process by providing the initial interval of interest. On this first interval, we compute Bernstein coefficients from the transformations presented in the previous section. Then in case of more that one sign change, we continue by invoking the splitting de Casteljau algorithm explained in the next subsection. 


\subsection{Splitting algorithm}

Given three pairwise distinct rational numbers $l, r, m$, there exists an efficient algorithm to deduce the two respective lists of Bernstein coefficients of a polynomial $p$ on intervals $(l, m)$ and $(m, r)$ from the list of Bernstein coefficients of $p$ on interval $(l, r)$.

Let $\mathrm{b}$ be the sequence of Bernstein coefficients of a polynomial $p$ of degree $n$ for an interval $(l, r)$. Let $m$ be a number distinct from $l$ and $r$. We pose $\alpha=\frac{m-l}{r-l}$ and $\beta=\frac{r-m}{r-l}$. The de_casteljau algorithm is defined recursively by:

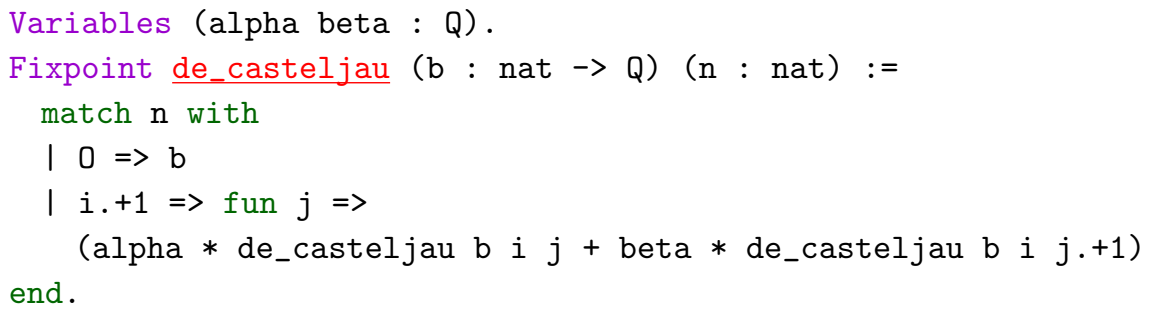

where the initial sequence of coefficients $b$ is represented by an infinite sequence of rational numbers, for which only the first $n$ elements are relevant. The following function gives the Bernstein coefficients of $p$ on the finite interval $(l, m)$.

Definition dicho' alpha beta c i :=

de_casteljau alpha beta c i 0 .

The following function gives the Bernstein coefficients of $p$ on the finite interval $(m, r)$.

Definition dicho alpha beta $\mathrm{p} c$ i :=

de_casteljau alpha beta $c(p-i) i$.

Observing the function de_casteljau more precisely, we see that the algorithm actually proceeds by creating a succession of lines where the element at rank $j$ in a given line is obtained by computing a weighted sum of the two elements at rank $j$ and $j+1$ on the previous line.

This process can be illustrated geometrically by a succession of broken lines. For the first line, we take the control line of the initial interval. Then, for each of the segments that compose this control line, we cut this segment in the same proportion as the proportion in which the interval is split between $(l, m)$ and $(m, r)$. This gives us a new collection of points. We started with $n+1$ control points and thus had $n$ segments, we now have $n$ new points, defining $n-1$ new segments. We repeat this process with the new segments, until we reach a situation where there is only one segment and we again split this segment into two parts in proportion of $(l, m)$ and $(m, r)$. The last point is guaranteed to lie on the polynomial's curve.

Although we actually only use de Casteljau's algorithm when $m$ is the midpoint of the initial interval, it works for any relative positions of $l, m$, and $r$, as long as they are pairwise distinct.

The different points computed by the de Casteljau algorithm are represented on Figure 9. The innermost points are the control points in the two new bases, computed from the original control points $\{B 0, \ldots, B 5\}$. The middle innermost control point, on 
the curve, belongs to the two new lists of control points. Points $\{C 0, \ldots, C 5\}$ are the control points in the left half, $\{D 0, \ldots, D 5\}$ are the control points on the right half. De Casteljau's algorithm is extensively used in computer graphics for rasterizing Bézier curves.

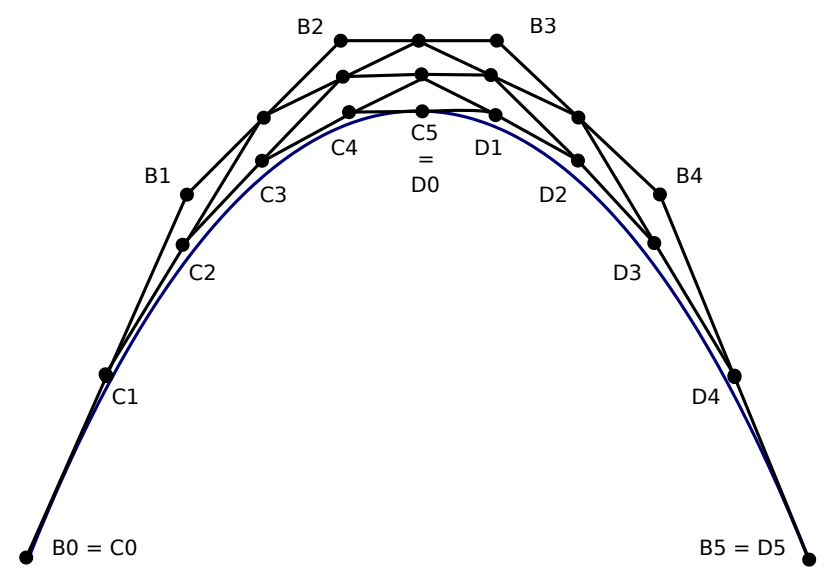

Fig. 9. Intermediate points computed by de Casteljau's algorithm on $[B 0, B 5]$

The aim of this section is to prove that this algorithms is correct, i.e. that the dicho and dicho' function indeed computes the expected Bernstein coefficients. The correctness theorems as stated in COQ are:

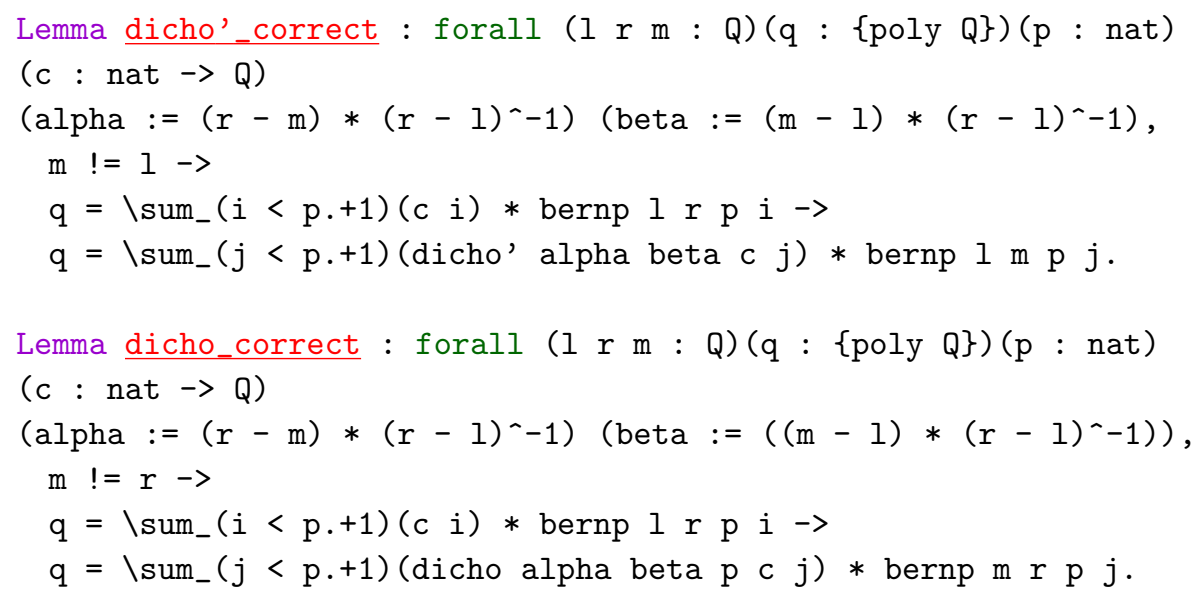

where (bernp 1 r p i) is the $i$-th polynomial in the Bernstein basis of degree $p$ with parameters $l$ and $r$.

The properties of computations performed by the de Casteljau algorithm are summarized on Figure 10. Starting from the input list $b=\left(b_{0}^{(0)} \ldots b_{p}^{(0)}\right)$ of coefficients in the basis with parameters $l$ and $r$, on the upper side of the triangle, it computes the full triangle, so that in the end the two expected output lists can be read on the two other sides of the triangle. The list $b^{\prime}=b_{0}^{(0)} \ldots b_{0}^{(j)} \ldots b_{0}^{(p)}$ is the list of coefficients in the basis 


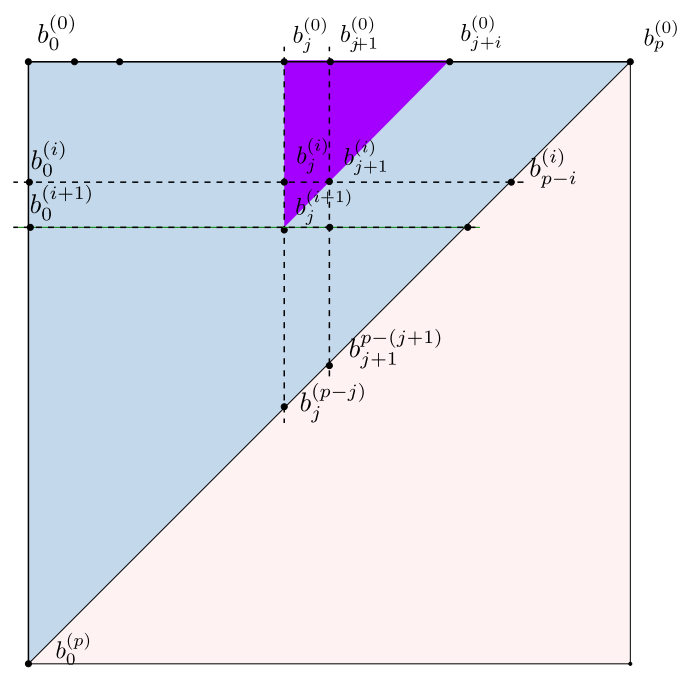

Fig. 10. Properties of de Casteljau computations

with parameters $l$ and $m$ output by dicho'. The list $b^{\prime \prime}=b_{0}^{(p)} \ldots b_{j}^{(p-j)} \ldots b_{p}^{0}$ is the list of coefficients in the basis with parameters $m$ and $r$ output by dicho. The small triangle area on Figure 10 shows which values the computation of an arbitrary given point in the triangle relies on. This structure is imposed by the fixpoint equation of the recursive definition of the de Casteljau algorithm:

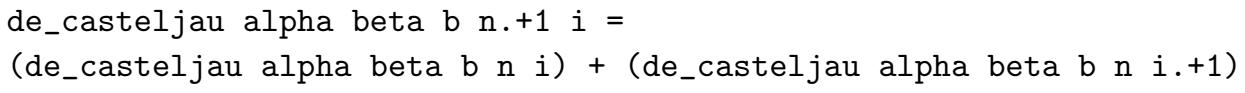

which looks very similar to the recursive relation governing the Pascal triangle.

Let us first notice that the shape of Bernstein polynomials implies that:

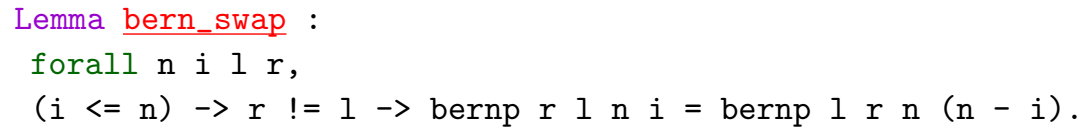

This remark implies that if $b$ is the list of coefficients of the polynomial $p$ in the Bernstein basis of degree $n$ with parameters $l$ and $r$, then the reverse of $b$ is the list of coefficients of the same polynomial $p$ in the Bernstein basis of degree $n$ with parameters $r$ and $l$ :

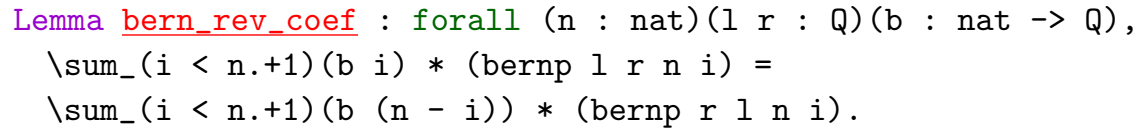

This remark shows that the correctness of the dicho' function is enough to get a certified computation of both Bernstein coefficient lists. If $b$ is the initial list of Bernstein coefficients with parameters $l$ and $r$, then reversing $b$ gives the coefficients with parameters $r$ and $l$. Applying dicho' on the reverse of $b$ using $r, l$, and $m$ computes the coefficients with parameters $r$ and $m$, hence reversing this output gives the result expected for dicho 
on $b$ using $l, r$, and $m$. Using a similar symmetry on the de_casteljau algorithm, we in fact reduce the proof of the dicho_correct specification to the proof of dicho'_correct.

By linearity, we can also reduce the proof of the dicho'_correct specification to the case where the input polynomial $p$ is in fact itself a Bernstein polynomial. This means that the input coefficient list $b$ only contains zeros except at one position where the coefficient is one.

Let us first compute the expected output of the dicho' function on such a list. In other words, for any distinct rational numbers $l, r, m$ and any $n \in \mathbb{N}$, given $i \leq n$, we want to compute the coefficients of:

$$
P_{b}(n, l, r, i)=\left(\begin{array}{c}
n \\
i
\end{array}\right) \frac{(X-l)^{i}(X-r)^{n-i}}{(r-l)^{n}}
$$

in the basis $\left(P_{b}(n, l, m, i)\right)_{i=0, \ldots, n}$. We pose $\alpha=\frac{r-m}{r-l}$ and $\beta=\frac{m-l}{r-l}$. In the polynomials of the new basis, formal denominators are of the form $(m-l)$. By noticing that:

$$
\frac{X-l}{r-l}=\beta \frac{X-l}{m-l} \quad \text { and } \quad \frac{r-X}{r-l}=\alpha \frac{X-l}{m-l}+\frac{m-X}{m-l}
$$

and by using the binomial identity:

$$
\left(\begin{array}{l}
n \\
i
\end{array}\right)\left(\begin{array}{l}
n-i \\
j-i
\end{array}\right)=\left(\begin{array}{l}
j \\
i
\end{array}\right)\left(\begin{array}{l}
n \\
j
\end{array}\right)
$$

we obtain that:

$$
P_{b}(n, l, r, i)=\sum_{j=i}^{n}\left(\begin{array}{l}
j \\
i
\end{array}\right) \alpha^{j-i} \beta^{i} P_{b}(n, l, m, j)
$$

Now to achieve the proof of the dicho'_correct lemma, it is sufficient to prove that the values output by the dicho' function coincide with the ones of $(*)$, which boils down to an induction on $i$.

\section{Formalization issues}

7.1. Sequences, iterations, polynomials

One of the key features of SSREFLECT libraries is that they devote a substantial effort to infrastructure. For instance the library about sequences contains as many operators as one would expect from a functional language standard library: factories, access, filtering, surgery...plus a comprehensive set of specifications for these operators. For instance, to construct the subdivision used in the proof of the weak intermediate value theorem proposed in section 2.5, it is enough to use the following tactic line in the proof:

pose $s l:=\operatorname{map}($ fun $\mathrm{k}=>\mathrm{x}+(\mathrm{y}-\mathrm{x}) *(\mathrm{k} \%: \mathrm{R} /(\mathrm{n} .+1 \%: \mathrm{R})))$ (iota $0 \mathrm{n} .+2)$.

The (iota $0 \mathrm{n} .+2$ ) operator constructs the sequence of integers between 0 and n.+2. Then the map generic list operator maps this sequence to the desired subdivision of the interval $[x, y]$. The infix,$+ *$ and $\backslash$ operators are the generic notations respectively for addition, product and division in a field, here used for the ordered archimedean field that is a parameter to the development. The $\%: \mathrm{R}$ annotation is a postfix notation for 
the embedding of integers in any ring structure (see section 7.2). Now the index of the first point in this sequence where the polynomial has a negative value is found by the following definition:

pose a'_index := find (fun $x=>$ p. $[x]>=0$ ) sl.

where again the find operator is a generic construction. The theory available on $f$ ind is sufficient to prove all the results needed in the proof.

In the SSREFLECT distribution, the sequence library is extended with a library about iterated operations (BGOP08). It provides a comprehensive infrastructure to work with the finite iteration of operators equipped with some known properties like associativity, commutativity or distributivity. Although the definition of iteration is naturally based on a sequence, the library includes a variety of indexing facilities. This library is crucial in the proof of the de Casteljau algorithm presented in section 6.3. both for the initialization with Cauchy bounds and for proving the correctness of the computation, that is that the output coefficients indeed provide a correct decomposition of the initial polynomial on the Bernstein basis. The \sum operator implicitly carries the properties that the iterated operation is commutative, associative, and that there is a product law which distributes over the sum. The sum_ $_{-}(i<n)$ notation indicates that the iteration is performed on the sequence of integers from 0 to n.-1 (meaning the sum might be empty). Manipulations of these expressions are treated in the generic theory of iterated operators.

Sequences are also the core of the definition of polynomials. The definition given in section 2.3 however provides little facilities to define a polynomial extensionally, by simply providing the values of its coefficients. This is made possible by the following construction, and its associated notation:

Notation "\poly_ $(i<n)$ E" := (Poly (mkseq (fun $i$ : nat $=>E$ ) $n)$ ).

The expression (mkseq ( $f$ un $i$ : nat $\Rightarrow \mathrm{E}$ ) $\mathrm{n}$ ) builds a sequence containing the $\mathrm{n}$ first values of the function $E$ over natural numbers thanks to the generic mkseq operator. The variable $i$ is bound in the body expression E. The Poly function of type

forall $\mathrm{T}$, seq $\mathrm{T} \rightarrow$ poly $\mathrm{T}$ normalizes the sequence it takes as argument into a polynomial, so that the tail zeroes are erased and a proof of normal form is added to the obtained sequence. Hence the size of the (sequence contained in the) polynomial $\backslash$ poly $(i<n) E$ is only smaller or equal to $n$, but is only equal to $n$ if $E n .-1$ is known to be non zero. This construction is used to define the expansion and translation introduced in section 5 , for instance:

Definition expand ( $\mathrm{p}:\{$ poly $\mathrm{Q}\})(\mathrm{k}: \mathrm{Q}):=$

$\backslash$ poly_ $(i<\operatorname{size} p)\left(p^{\prime} \_i * k^{`}+i\right)$.

where $Q$ is the ordered archimedean field parameterizing the development and ${ }^{+}+$is the exponentiation operation (see section 7.2). These definitions are accompanied by correctness lemmas in term of evaluation, like:

Lemma eval_expand : forall $\mathrm{pkx}$, (expand $\mathrm{p} \mathrm{k}$ ). [x] $] \mathrm{p} \cdot[\mathrm{k} * \mathrm{x}]$.

where $\mathrm{p} .[\mathrm{x}]$ denotes the evaluation of the polynomial $\mathrm{p}$ at the point $\mathrm{x}$. 
The list of coefficients defining a polynomial is of course the coefficients of the polynomial in the (countable) infinite basis of monomials. In this development, we also need to consider polynomials as vectors in the vector space of polynomials of degree less than a fixed value, again with coordinates in the basis of monomials or in Bernstein bases. More precisely we only use the fact that monomials and Bernstein polynomials are two families of generators and not their linear independence. Unfortunately, the linear algebra part of the SSREFLECT archive is not yet sufficiently well integrated to get this easily from an existing infrastructure. We hence only define these linear algebra operations at a low level: the list of coefficients defining a polynomial is padded with zeroes when the list of coefficient in a monomial basis of a certain degree is needed, and conversely the list of coefficients in a monomial basis needs to be normalized when we need to go back to the actual polynomial. It would certainly be more elegant to use a generic library than to rely on this ad-hoc solution.

\subsection{Algebraic structures}

This formalization is structured along the algebraic hierarchy proposed by the SSREFLECT libraries $\left(\mathrm{GMR}^{+} 07\right)$. This hierarchy builds a graph of algebraic structures based on types with decidable equalities and implement inheritance and sharing or theories and notations through the canonical structures type inference mechanism of the CoQ system. We moreover base our proofs on an extension of this hierarchy for ordered structures (Coh10). This extension allowed to consider ordered unit rings and fields, which are unit rings and fields equipped with a decidable (total) boolean order relation which is compatible with addition and product. In the proofs we describe here, the main algebraic structures involved are the field of coefficients and the commutative ring structure that polynomials inherit. This inheritance is completely automated by the infrastructure already present in the SSREFLECT libraries. However natural numbers are not equipped with any such algebraic structure in the SSREFLECT library and their theory and notations are defined separately. We can still use the same symbols for their algebraic operations and ordering thanks to the scoping mechanism of the CoQ system (Coq), even if this sometimes require an explicit scoping annotation in theorem statements.

Natural numbers are used to define the (discrete) exponentiation as iterated product in ring structures: if $\mathrm{x}$ belongs to a type equipped with a ring structure and ( $\mathrm{n}: \mathrm{nat}$ ) is a natural number, $\mathrm{x}^{\wedge}+\mathrm{n}$ denotes $\mathrm{x}$ to the $\mathrm{n}$-th power, i.e. $\mathrm{x} *(\mathrm{x} * \ldots(\mathrm{x} * \mathrm{x}) \ldots)$. Since at the time we write these lines the SSREFLECT libraries do not feature a formalization of integers, exponentiation in a field structure can become artificially technical: $x^{\wedge}-n$ only denotes the inverse of $x^{\wedge}+n$, with a (positive) natural number exponent. Yet for this work this defect was not a severe limitation. An other interesting operation is the iteration of the addition operation in a ring structure: $\mathrm{x} *+\mathrm{n}$ denotes $\mathrm{n}$ times $\mathrm{x}$, i.e., $\mathrm{x}+(\mathrm{x}+\ldots(\mathrm{x}+\mathrm{x}) \ldots)$. In a commutative ring structure, the expression developing the power of a sum is hence:

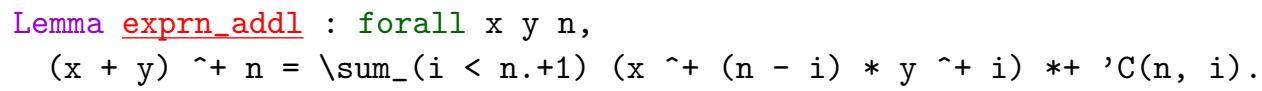


as available in the SSREFLECT library. This allows to combine smoothly the binomial identities also available in SSREFLECT with operations in a commutative ring, as necessary in the correctness proofs of the de Casteljau algorithm presented in section 6.3. When the iterated element $\mathrm{x}$ is in fact 1 the unit element of the ring, this operation defines a generic image of the semi-ring of natural numbers in the ring. Note that this operation need not be injective: this will only be the case if the characterictic is zero. This embedding is so widely used that a new notation is defined: $\mathrm{n} \%$ : $\mathrm{R}$ denotes $1 *+\mathrm{n}$ in any ring structure. Remark that this expression needs a context or an explicit cast to determine the ring in which the natural number should be injected, i.e. the ring 1 is the unit of. This injection appears in particular in the hypothesis that the field is archimedean, which is necessary for the weak intermediate value theorem. This hypothesis is stated as follows:

Variable Q : oFieldType.

Hypothesis Q_arch : forall $\mathrm{x}: \mathrm{Q}, 0<=\mathrm{x} \rightarrow\{\mathrm{n}:$ nat $\mid \mathrm{x}<=\mathrm{n} \%: \mathrm{R}\}$.

where the Variable Q declares an ordered field structure parameter, and the Q_arch hypothesis gives access to the explicit value of an integer larger than a given element of the ordered field. Again, the SSREFLECT distribution lacks a library about integers and rational numbers, the manipulation of the embedding of rational numbers in this archimedean field is sometimes unnecessarily tedious, though again this was not really an issue in this development.

\subsection{Automation issues}

Sadly, a significant part of scripts is devoted to too many atomic rewrite steps to normalize ring expressions, or prove trivial consequences of the properties of order like transitivity or compatibility with field operations. The SSREFLECT libraries still lack the standard automated proof producing decision procedures available in the CoQ system, like ring normalization or linear arithmetic decision. The SSREFLECT structures are indeed still not connected to these mechanisms. The CoQ tactics on linear arithmetic are hardcoding the representation of integers and coefficients and should rely on a more abstract structure like the one of ordered field. In the case of the automation of ring identities, it would significantly help the user if normalization could handle simultaneously the various ring and semi-ring structures that can occur in an expression, like the rings of polynomial coefficients, polynomials themselves and possibly the semi-ring of natural numbers. In the case of the automation of ordered arithmetic, proof steps often involve non linear expressions, for which it is quite difficult to get a truly generic and efficient proof producing decision procedure. This is in fact part of the long term objectives of this work, namely to certify a complete decision procedure for the full first order theory of real closed fields. Yet incomplete but lightweight tools could probably be crafted to relieve the user from tedious steps when possible. 


\subsection{Current state of the formalization}

In this section, we recapitulate the main results described in this paper that have a formal proof in our development.

- The absolute values of the real roots of a polynomial are bounded by the Cauchy bound, which is expressed only using the absolute values of the coefficients of the polynomial.

- If a polynomial function $p$ has a negative value in $x$ and a positive value in $y$, with $x<y$, then for any $\varepsilon$ one can exhibit $x^{\prime}$ and $y^{\prime}$ such that $-\varepsilon<p\left(x^{\prime}\right)<p\left(y^{\prime}\right)<\varepsilon$.

- If a polynomial only has one sign change in its coefficients for the standard monomial basis, then this polynomial has exactly one root between 0 (excluded) and $+\infty$.

- If a polynomial only has one sign change in its Bernstein coefficients for a given interval $(l, r)$, then this polynomial has exactly one root between $l$ and $r$ (excluded).

- The transformation that maps any polynomial to its Bernstein coefficients maps Bernstein polynomials to plain monomials.

- De Casteljau's algorithm computes correctly the Bernstein coefficients for the intervals $(l, m)$ and $(m, r)$ from the Bernstein coefficients for the interval $(l, r)$.

This work is part of a more ambitious plan, aiming at providing an efficient procedure to isolate the roots of any polynomial. It remains to develop the connections between the various results that will constitute this procedure and its proof of correctness. To certify an algorithmically naive version of such a procedure, we still need to describe the procedure to reduce any polynomial to a separable polynomial (a polynomial where all roots have multiplicity 1 ). The easy approach is to divide by the greatest common divisor between the polynomial and its derivative. The reduction to separable polynomials should not require too much effort considering the libraries already available in the SSREFLECT package. We also need to describe the termination of a procedure based on successive dichotomy. This study of termination might however require more substantial work.

Another issue will be to connect the correctness proof of such a naive implementation with more realistic programs, like an implementation of de Casteljau whose complexity would be linear in the degree of the input, as implemented in (Mah07) or even more optimized codes like the ones of (MRR05).

\section{Conclusion}

Real root isolation methods by sign-change-based methods is a classical topic, extensively

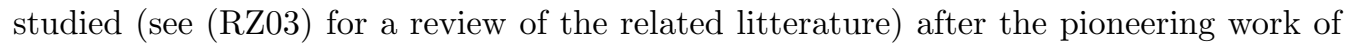
Uspensky (Usp48). Bernstein polynomials are used to provide efficient implementations of these methods (MRR05: RZ03). To our knowledge, this work is the first mechanized proof of de Casteljau's algorithm, and of the building blocks of a real root isolation procedure. The closest work to ours is probably the study of global optimization methods in CoQ lead by Roland Zumkeller (Zum08). Indeed, Bernstein polynomial bases are also used to approximate continuous functions on a closed domain. This last work results in an implementation in the CoQ system of a tool to bound optima of multivariate continuous 
real functions. Yet we could not find mention of a formalization of the correctness proofs of this tool.

Other work concerned with roots of continuous functions often relies on Newton's method. This method has also be described formally using CoQ and SSREFLECT, although in a less constructive setting (Paş10). However, the applicability of the work is quite different: the work in (Paş10) applies to a wide variety of functions of class $\mathrm{C} 2$ and it guarantees the unicity of a root in an interval using strong constraints on the first and second derivative of the function inside the interval. It does not provide any tool for the case when the chosen interval does not satisfy the conditions, while our work provides a study of a dichotomy approach, but is concerned solely with polynomials.

This work on Bernstein polynomials combines techniques coming from analysis, algebra, and geometry. For instance, the properties of reversing the list of coefficients of a polynomial are studied by looking at the polynomial as a function from rational numbers to rational numbers. Similarly, the proof of Descartes' law of signs works by looking at functions and bounds on their values in various intervals. On the other hand, the definition of Bernstein coefficients relies on concepts that come from linear algebra: vector spaces, bases, or morphisms. Last, de Casteljau's algorithm relies on geometry with midpoints or segments. It is particularly exciting that we can now study formally mathematical algorithms that use all these aspects of mathematics.

This development is not made just for the beauty of it. The initial goal is to provide one of the basic blocks required for cylindrical algebraic decomposition (BPR06, Mah07). In the short term, we want to complete this into a full algorithm to isolate the roots of an arbitrary polynomial. This involves proving the technique to reduce the multiplicity of roots that we already described in the introduction, initializing the search for roots with an interval large enough to contain all the roots, programming the recursive dichotomy process, and proving that this process always terminates.

For the proof of termination, we already know a mathematical argument, described in (BPR06) under the name "theorem of three circles". However, this theorem uses arguments based on complex numbers and we wish to find a more elementary proof, as we still want to express our result using mainly rational numbers. Our proof of the law of signs already is more elementary than the ones found in the literature.

In the long run, a good knowledge of Bernstein polynomials and coefficients opens the door to a wide variety of tools that are commonplace in computer aided design and robotics. Bézier curves which are often used in drawing tools share a lot of properties with Bernstein control points. Concerning robotics, splines and Bézier curves can also be used to describe the trajectory of moving vehicles. Thus, this work may eventually be useful for the formal verification of critical software in robotics.

\section{Acknowledgments}

The authors whish to gratefully thank the anonymous referees who have suggested numerous improvements both for the formalization and for its description. 


\section{References}

Yves Bertot and Pierre Castéran. Interactive Theorem Proving and Program Development, Coq'Art:the Calculus of Inductive Constructions. Springer-Verlag, 2004.

Pierre Bézier. Courbes et Surfaces. Hermès, 1986.

Yves Bertot, Georges Gonthier, Sidi Ould Biha, and Ioana Paşca. Canonical Big Operators. In Proceedings of the 21st International Conference on Theorem Proving in Higher Order Logics (TPHOLs 2008), volume 5170 of Lecture Notes in Computer Science, pages 12-16. Springer, August 2008. http://hal.inria.fr/inria-00331193/

Saugata Basu, Richard Pollack, and Marie-Françoise Roy. Algorithms in Real ALgebraic Geometry, volume 10 of Algorithms and Computations in Mathematics. Springer, second edition, 2006.

Luís Cruz-Filipe, Herman Geuvers, and Freek Wiedijk. C-CoRN, the Constructive Coq Repository at Nijmegen. In Andrea Asperti, Grzegorz Bancerek, and Andrzej Trybulec, editors, MKM, volume 3119 of Lecture Notes in Computer Science, pages 88-103. Springer, 2004.

Cyril Cohen. Formalizing real analysis for polynomials. Technical report, Inria, 2010. http: //hal.inria.fr/inria-00545778/en/

Coq team. The Coq System. http://coq.inria.fr/

Paul de Castleljau. Formes à pôles. Hermès, 1985.

René Descartes. Géométrie (1636). A source book in Mathematics. Harvard University Press, 1969.

Georges Gonthier and Assia Mahboubi. A Small Scale Reflection Extension for the Coq system. Research Report RR-6455, INRIA, 2008. http://hal.inria.fr/inria-00258384/en/

Georges Gonthier and Assia Mahboubi. An introduction to small scale reflection in Coq. Technical report, INRIA, 2010. to appear in Journal of Formal Reasoning.

Georges Gonthier, Assia Mahboubi, Laurence Rideau, Enrico Tassi, and Laurent Théry. A modular formalisation of finite group theory. In Klaus Schneider and Jens Brandt, editors, TPHOLs, volume 4732 of Lecture Notes in Computer Science, pages 86-101. Springer, 2007. Donald Knuth. Metafont: the Program. Addison Wesley, 1986.

Assia Mahboubi. Implementing the cylindrical algebraic decomposition within the coq system. Mathematical Structures in Computer Science, 17(1):99-127, 2007.

Mathematical Components team. The Ssreflect Distribution. http://www.msr-inria.inria. fr/mathcomp

Bernard Mourrain, Fabrice Rouillier, and Marie-Françoise Roy. Bernstein's basis and real root isolation. Mathematical Sciences Research Institute Publications, 2005.

Russell O'Connor. A monadic, functional implementation of real numbers. Mathematical Structures in Computer Science, 17(1):129-159, 2007.

Russell O'Connor. Certified exact transcendental real number computation in coq. In TPHOLs, Lecture Notes in Computer Science, pages 246-261. Springer, 2008.

Ioana Paşca. Formal proofs for theoretical properties of newton's method. Mathematical Structures in Computer Science, this issue, 2010.

Fabrice Rouillier and Paul Zimmermann. Efficient isolation of polynomial real roots. Journal of Computational and Applied Mathematics, 162(1):33-50, 2003.

Anne Sjerp Troelstra and Dirk van Dalen. Constructivism in Mathematics, an introduction, volume 1. North-Holland, Amsterdam, 1988.

James Victor Uspensky. Theory of Equations. MacGraw-Hill Bok Company, 1948.

Roland Zumkeller. Global Optimization in Type Theory. PhD thesis, École Polytechnique, 2008. 